\title{
El liderazgo brasileño en Sudamérica en tiempos de cambio \\ Una mirada desde Uruguay
}

\author{
A liderança brasileira na América do Sul em tempos de mudança \\ Um olhar a partir do Uruguai
}
The Brazilian leadership in South America in times of change A view from Uruguay

\begin{abstract}
Resumen: Desde el análisis de las preferencias de los legisladores uruguayos, este trabajo reflexiona sobre la influencia que Brasil ejerce sobre los países de la región y el liderazgo que proyecta. Para realizar este ejercicio se encuestó al 96\% del Parlamento de Uruguay, se analizó la prensa, el debate parlamentario durante el período (20102015), y se realizaron entrevistas a informantes calificados y actores clave. A partir de la información recabada y partiendo del análisis del caso uruguayo, se realiza un ejercicio de construcción de preguntas e hipótesis sobre el impacto que la actual crisis interna brasileña y la reconfiguración del escenario mundial pueden tener en su accionar internacional y en las políticas exteriores de los países sudamericanos.
\end{abstract}

Palabras clave: Política exterior uruguaya. Liderazgo brasileño. Partidos políticos. Uruguay.

Resumo: A partir da análise das preferências dos legisladores uruguaios, este trabalho reflete a influência que o Brasil exerce sobre os países da região e a liderança que ele projeta. Para realizar este exercício, foi analisado um survey de $96 \%$ do Parlamento do Uruguai, a imprensa, o debate parlamentar durante o período (2010-2015) e foram realizadas entrevistas com informantes qualificados e atores-chave. Com base nas informações recolhidas e com base na análise do caso uruguaio, um exercício é realizado para construir questões e hipóteses sobre o impacto que a atual crise interna brasileira e a reconfiguração do cenário mundial podem ter sobre suas ações internacionais e as políticas externas dos países sul-americanos.

Palavras-chave: Política externa uruguaia. Liderança brasileira. Partidos políticos. Uruguai.

Abstract: Based on an analysis about the preferences of the Uruguayan legislators, this work analyzes the Brazilian influence on the countries of the region and the leadership that it projects. The information and database for this analysis comes from a survey of

\footnotetext{
* Facultad de Ciencias Sociales de la Universidad de la República (Udelar, Montevideo, Uruguay).

autor.datos_biográficos
}

Civitas, Porto Alegre, v. 18 , n. 2, p. 376-392, mayo-agosto 2018

Exceto onde especificado diferentemente, a matéria publicada neste periódico 
$96 \%$ of the Uruguayan parliament, the analysis of the parliamentary debate in the period (2010-2015) and the press, and interviews of qualified informants and key actors. From the information gathered and based on the analysis of the Uruguayan case, this study proposes an exercise of formulating questions and hypotheses about the impact that the Brazilian's current internal crisis and the reconfiguration of the international system may have had on both on its international action and in the Southamerican countries' foreign policies.

Keywords: Uruguayan foreign policy. Brazilian leadership. Political parties. Uruguay.

\section{Introducción}

Este trabajo busca analizar las percepciones de los parlamentarios uruguayos sobre la influencia que Brasil ejerce sobre los países de la región. Y a partir del estudio de las preferencias de los legisladores uruguayos, se realiza un ejercicio de construcción de preguntas e hipótesis sobre el impacto que la actual crisis interna brasileña y la reconfiguración del escenario internacional pueden tener en su accionar internacional y en las políticas exteriores de los países sudamericanos, partiendo del análisis del caso uruguayo. ${ }^{1}$

La influencia, o el liderazgo regional brasileño, puede disminuir por desatención de la región en un futuro próximo y por tanto las expectativas sobre Brasil de las élites políticas de los países de la región pueden verse frustradas. $\mathrm{O}$ un giro en la política exterior de Brasil podría convertirlo, por subordinación, en un dinamizador de un redireccionamiento de las políticas exteriores sudamericanas hacia los Estados Unidos de América (EEUU) en función del escenario resultante de la actual situación brasileña. Asumir una u otra posición terminaría generando, probablemente, cambios entre las valoraciones positivas o negativas que sobre Brasil existen, con una posible y curiosa inversión de las mismas.

De mantenerse la actual divergencia con las orientaciones estadounidenses en materia internacional, la escasez de recursos internos que se pueden volcar en Brasil hacia el accionar exterior podría llevar al primero de los escenarios planteados con consecuencias fuertes de desgaste de la imagen de Brasil en la región. Mientras que si persiste la voluntad de mantener un protagonismo internacional, aunque sea de segundo orden, la carencia de recursos y por tanto de poder endógenamente construido pueden llevar a Brasil, de la mano se la conducción profesional de Itamaraty, a ponerse bajo la tutela de los EEUU,

\footnotetext{
${ }^{1}$ El presente trabajo recoge algunas reflexiones previas de los autores realizadas en Luján (2016) y López Burian (2015 y 2016).
} 
rompiendo parte de las alianzas con el Sur emergente, y consolidando una lógica clásica de esferas de influencia a nivel continental o más precisamente hemisférico. Entre el Sur y el Norte la elección para Brasil podría ser Occidente. Dos interrogantes surgen del análisis de dichas situaciones: ¿cómo se posicionarían las élites de nuestros países en general ante tal orientación de la política exterior brasileña? Y en particular, ¿cómo lo harían las de Uruguay?

En el presente trabajo luego de presentar la literatura que analiza el liderazgo regional brasileño, la política exterior de este país y la política exterior uruguaya, se describe el abordaje metodológico utilizado para la recolección de datos para el análisis de las preferencias de los legisladores uruguayos en materia de política exterior. Posteriormente se analiza el caso uruguayo, con el fin de proponer un conjunto de reflexiones sobre los escenarios futuros que se podrían presentar en el liderazgo brasileño y sus impactos en las políticas exteriores de los países sudamericanos.

\section{EI liderazgo brasileño y la influencia sobre Uruguay: la literatura sobre dos políticas exteriores y sus interacciones}

Este apartado, tiene como objetivo presentar una serie de debates presentes en la literatura sobre la política exterior brasileña y uruguaya, con el fin de ubicar el asunto central del trabajo que es analizar las percepciones de los parlamentarios uruguayos sobre la influencia que Brasil ejerce sobre los países de la región y particularmente sobre Uruguay.

Desde fines del siglo XX, Brasil fortaleció una estrategia de proyección de su influencia sobre su entorno cercano y definió a su espacio geográfico regional como América del Sur, alejándose del horizonte latinoamericano donde México es su gran competidor, el cuál luego de tener una fuerte convergencia con EEUU, se ve enfrentado a su vecino del norte desde la asunción de Donald Trump como presidente. Es, pues, México una potencia hemisférica de tamaño medio competidora directamente de Brasil en la región latinoamericana.

Esta definición de región y la proyección de su influencia fueron acompañadas desde la literatura especializada por un debate sobre el liderazgo regional brasileño. La conceptualización de Brasil como paymaster del proceso de integración regional tiene interpretaciones diferentes. Hay quienes sostienen que Brasil no cumple este papel por razones vinculadas a sus elecciones y apuestas, mientras otros cargan las tintas en dificultades estructurales de fondo, económicas y políticas (Vigevani et al., 2008) Parte de estos trabajos afirman la existencia de lo mismo (Saraiva, 2010; Pinheiro y Gaio, 2014), partiendo 
de la idea de un Brasil que acepta cargar con los costos de este liderazgo y a su vez propone que esta posición es aceptada por el resto de los países sudamericanos. Frente a este argumento existen diferentes cuestionamientos que señalan las dificultades de Brasil para liderar la región (Flemes, 2009; Burges, 2015; Flemes y Wehner, 2015).

Por una parte, algunos cuestionan el liderazgo brasileño señalando la falta de capacidades como el factor clave (Flemes, 2009), mientras otros consideran que Brasil vive una crisis de liderazgo en su ámbito de influencia, desde el fin del segundo gobierno de Lula (Burges, 2015). Desde un enfoque realista, la construcción del liderazgo brasileño no colocó enfáticamente en el centro de su estrategia a los aspectos clásicos de poder político (Hurrell, 1998), posicionándose como "hegemón consensual" que busca conjugar intereses de varios actores. Por otra parte, otros académicos argumentan que el liderazgo brasileño no es una realidad incontestada o incontestable, ya que otras potencias medias pueden desafiar su posición en la región (Flemes y Wojczewski, 2010). En este sentido, Andrés Malamud (2011), partiendo de la existencia de posibles rivales y el déficit relativo de recursos, caracterizó a Brasil como un "líder sin seguidores", o que primero busca ser un jugador global, aunque sea de los de menor peso relativo, antes que buscar contar con el reconocimiento de su liderazgo por parte de los demás países sudamericanos para desde allí proyectarse al escenario mundial.

Por otra parte, la literatura sobre la política exterior uruguaya ha señalado la existencia, desde los tiempos de las guerras civiles post independencia, de una política pendular entre Argentina y Brasil (Pérez, 2011, p. 9-10). Esta dinámica en la orientación de la política exterior recoge dos componentes que orientan su acción, uno que se vincula con las relaciones políticas de los partidos desde sus procesos genéticos, y otro elemento de tipo pragmático signado por la coyuntura (Caetano et al., 2016. Aunque algunos autores señalan que el vínculo estratégico con Brasil tiene larga data y se mantuvo estable en términos relativos (Caetano, 2012; Alvariza y Clemente, 2014), a la alianza estratégica de Uruguay durante la presidencia de José Mujica y el alejamiento de Argentina desde la primera presidencia de Tabaré Vazquéz marcan un momento de cercanía del péndulo con respecto a Brasil. La actual administración, la segunda presidencia de Tabaré Vazquéz, ha dado muestras de que el ciclo pendular ha seguido su curso histórico de acercamiento a la Argentina, Diana Tussie y Nicolás Comini así lo reseñan al destacar que "[...] en la práctica hay un viraje en el tratamiento a las plantas productoras de celulosa [...] se derogo la disposición 1108/13 de la Secretaría de Puertos y 
Vías Navegables, la cual impedía que las cargas de exportación con origen argentino fueran transbordadas en el puerto de Montevideo" (Tussie y Comini, 2016, p. 12-13); a lo que agregan que la autoridades argentinas "[...] se expresaron en favor de llevar un acuerdo Mercosur UE" (Tussie y Comini, 2016, p. 13).

Adicionalmente, es clave señalar algunos aspectos del relacionamiento entre EEUU y Brasil. Desde inicios del siglo XX, Brasil comenzó su acercamiento a EEUU a través de una estrategia de "bandwagoning", siendo esta la más frecuente de Brasil durante el siglo XX (Rodriguez, 2015, p. 376). Por su parte, Maria Regina Soares Lima señala que hasta inicios de la década de 1960 la política exterior brasileña se orientó a partir del paradigma "americanista", que colocaba a EEUU como eje de dicha política, al ser visto como una potencia hemisférica y global (Lima, 1994, p. 35). Leticia Pinheiro (2000 y 2004) también identifica la influencia del "americanismo", al cual le siguieron etapas caracterizadas por orientaciones diferentes, dos etapas "globalistas" y una "institucionalista pragmática", donde Brasil desde los años noventa del siglo XX promueve los acuerdos regionales y la integración pero no apuesta decididamente a profundizar las instituciones para mantener libertad de acción (Pinheiro, 2000, p. 325-326).

Esta convergencia con EEUU es recogida por la literatura especializada en la política exterior brasileña, aunque se señalan búsquedas de autonomía que varían en diferentes momentos del siglo XX. Amado Cervo (2003 y 2005) construye un análisis de la política exterior brasileña a partir de la coexistencia y el diálogo de dos modelos desarrollistas, uno de los cuales veía a EEUU como el vínculo privilegiado con las fuerzas del capitalismo externas al país. Gelson Fonseca (2004, p. 362) coloca el relacionamiento con este país en el marco de la búsqueda de autonomía y señala la distancia que Brasil toma de la potencia norteamericana a fines de los setenta tanto en lo militar ${ }^{2}$ como en lo económico. A la búsqueda de "autonomía por la distancia" le siguió una "autonomía por la participación", donde Brasil subrayó en su agenda el Consejo de Seguridad de la ONU, las Operaciones de mantenimiento de la Paz de ONU (OMP) e intensificó el diálogo con Europa como forma de búsqueda de la misma.

Ese distanciamiento de Brasil en relación a EEUU, abordado a través de narrativas históricas (Moniz Bandeira, 1986), es comprobado a partir de la utilización de métodos estadísticos por parte de Octavio Amorim Neto (2012). En su análisis del período 1946-2008, Brasil transita de una estrategia

\footnotetext{
${ }^{2}$ En 1977, Brasil rompe su acuerdo militar con EEUU.
} 
de "bandwagoning" a un "soft-balancing", consonante con el "revisionismo moderado" de la orientación de la política exterior brasileña señalado por María Regina Soares Lima (1994). Las relaciones entre ambos países despertaron el interés de varios académicos (Almeida y Barbosa, 2006; Moniz Bandeira 1986, 2003, 2004 y 2010 y Silva y Spohr 2015). Monica Hirst (2009) también consigna el alineamiento con EEUU hasta fines de la década de 1960. Luego de un período de autonomía, con la vuelta a la democracia Brasil tiene nuevo un acercamiento con la potencia hemisférica, señalando que los gobiernos de Lula oscilaron entre la continuidad y el cambio, predominando la primera (Hirst, 2009, p. 153). Amorim Neto en las conclusiones de su trabajo señaló que las transformaciones en el escenario mundial a partir de 2003 -aceleradas desde la crisis de 2008- junto a los cambios domésticos hacían muy probable que los determinantes de la política exterior brasileña cambiasen (2012, p. 177). En este sentido Janina Onuki y Amâncio de Oliveira (2010) señalan que el análisis de las diferencias en la orientación de la política exterior, como variable dependiente, debe concentrarse en si el relacionamiento privilegia relaciones Norte-Sur o Sur-Sur y la actitud de los actores frente a la integración regional.

Esto hace que, en un escenario que se reconfigura, otros actores sean desafiantes para el liderazgo regional brasileño. China con quien comparte múltiples foros internacionales, de los llamados "países emergentes", es un actor con creciente peso en la región desde hace ya un tiempo considerable (Dosch y Goodman, 2012). Este país se ha constituido en un competidor para Brasil en su proyección atlántica (Urdinez, 2014) y también particularmente sobre su influencia en la región (Jenkins, 2014; Urdinez et al,, 2016) a la vez que cuenta con capacidades para establecer acciones económicas con sus socios que impactan positivamente en su interés de fortalecer las relaciones bilaterales con países de la región (Strüver, 2014).

\section{Análisis empírico}

\section{Fuentes y datos}

Para obtener datos primarios sobre las percepciones de los legisladores uruguayos en relación a la influencia de Brasil en la política exterior uruguaya, se encuestó a los parlamentarios uruguayos de la pasada legislatura (el universo consta de 99 diputados, 30 senadores y el Vicepresidente de la República, quien por disposición constitucional preside el Senado y la Asamblea General). La encuesta cubrió el 96\% del universo, no existiendo desbalances significativos entre partidos ni entre los sectores intrapartidarios. 
Adicionalmente se realizaron 51 entrevistas en profundidad, no estructuradas, a políticos e informantes calificados.

\section{Análisis}

Como se ha planteado, el liderazgo brasileño enfrenta los desafíos de su situación doméstica y el impacto de sus competidores. Sean, en esta última dimensión, la influencia de China o EEUU a nivel global o México y Argentina a nivel regional o subregional. Para analizar las preferencias de los legisladores uruguayos, este apartado se ocupa en explorar las percepciones sobre los actores internacionales antes mencionados, sobre sus relaciones con Uruguay, sobre las expectativas de su relacionamiento, sobre su disposición en ampliar el relacionamiento comercial, sobre la influencia que ejercen y los matices que los cambios en las orientaciones de los actores pueden generar en los actores uruguayos.

\section{Cuál es la región o cuáles son los polos del péndulo}

Una primera aproximación posible sobre el foco territorial prioritario es ver las preferencias que los actores tienen sobre los regímenes internacionales en tanto entramados institucionales. Para ello se consultó a los legisladores sobre a qué instituciones se debería apostar a fortalecer a través de la política exterior del país. La Tabla 1 nos proporciona la información referida específicamente al partido de gobierno (Frente Aamplio) y su discriminación por alas de lo mismo.

Tabla 1. Institución aproximada a la idea de región a la priorizada en política exterior por los legisladores del Frente Amplio

(respuestas segmentadas por ala de partido).

\begin{tabular}{llcc}
\hline Ala & & Frec. & \% \\
Ala izquierda FA & Mercosur & 3 & 10,7 \\
& Unasur & 18 & 64,3 \\
& Celac & 7 & 25,0 \\
& Total & 28 & 100,0 \\
Ala derecha FA & Mercosur & 10 & 43,5 \\
& Unasur & 11 & 47,8 \\
& Celac & 2 & 8,7 \\
& Total & 23 & 100,0 \\
\hline
\end{tabular}

Fuente: Elaboración propia a partir de encuesta realizada a legisladores uruguayos. 
En términos generales, se percibe una talante sudamericanista en todos los legisladores frenteamplistas, pero mientras que los de su ala izquierda casi en dos terceras partes abrazan la institucionalidad unasuriana como la preferida, no llegan a la mitad las preferencias por la Unasur entre los que integran el ala derecha del FA, siendo entre ellos muy fuerte la comarca más próxima, el Mercosur, como la región priorizada, por último se debe destacar que el espacio regional visto en su máxima amplitud (América Latina y el Caribe) sólo es visto como la región a priorizar por uno de cada cuatro legisladores del ala izquierda del Frente Amplio y uno de cada once de los restantes, lo que muestra diferencias con una historia de latinoamericanismo muy fuerte. Esta retracción podría ser coyuntural, al "achicarse" la región hacia una Sudamérica más homogénea y cercana.

Latinoamérica y el Caribe es el espacio donde EEUU despliega su capacidad de influencia regional y donde Brasil se encuentra con su principal competidor, en dicho ámbito: México. Unasur, como columna vertebral del proyecto continental brasileño, particularmente luego del pasado "giro a la izquierda" de la región, es valorado negativamente por los partidos de derecha y centro derecha, tanto por la confluencia de gobiernos de izquierda o progresistas en torno al proceso, como por el vector político que estructura a este proceso. Un senador de la oposición, al ser entrevistado, visualizó al espacio de la Alianza del Pacífico como una dimensión donde "pendular" entre México y Brasil como clave táctica de la política exterior a partir de una reconfiguración del espacio regional, y donde Argentina ha perdido peso en los últimos años:

Uruguay construyó su política exterior pendulando entre Argentina y Brasil, porque eran países similares en su peso. Argentina se desplomó y Brasil es potencia. El riesgo de Uruguay es quedar adosado a Brasil. Por eso la Alianza del Pacífico es una oportunidad para vincularse con el nuevo eje mundial y de tener otro polo para pendular, México y sus aliados. [...] Por eso la UNASUR es un invento brasileño contra México. A Brasil hay que aprovecharlo y tener buenas relaciones [...] pero no terminar en una situación de total dependencia a la que estamos encaminados. [...] Brasil es una ventana al mundo y México puede ser otra.

El actual giro a la derecha de la región en general y de la Argentina y Brasil en particular, tanto en sus políticas domésticas como en sus políticas internacionales, seguramente están realineando las posturas de las élites políticas más conservadoras del Uruguay, aperturistas por definición y por tanto con miradas sobre la inserción internacional del país más cercanas a las de sus pares de los países vecinos. El elenco gobernante hoy en Uruguay, 
ideológicamente pragmático y de centro-izquierda, en materia internacional continua apostando a la integración regional siempre y cuando la misma permita avanzar en acuerdos como el que se está negociando con la UE, base de otros futuros tratados de libre comercio con otras potencias del mundo desarrollado o emergentes.

\section{La coyuntura regional e internacional: la mirada sobre el afuera}

Las configuraciones del sistema internacional, los procesos regionales, las coyunturas específicas en estos escenarios, la interacción entre los actores, los recursos de poder de los mismos, los hechos concretos (como las crisis o las guerras) son claves pues se transforman en variables contextuales que pueden transformarse en intervinientes en la política exterior de un país. Pero, siguiendo a Miriam Saraiva y Laura Tedesco (2004), no basta con quedarse solamente con hechos concretos, ya que las percepciones de los actores sobre el escenario externo importan y mucho.

Consultados sobre la influencia ${ }^{3}$ de las dinámicas globales y regionales, los legisladores uruguayos tomados como un único conjunto manifestaron que los procesos del ámbito del Mercosur son los que más influyen la política exterior uruguaya. La dinámica del sistema internacional es percibida como influyente, superando la mitad de la escala, pero un punto menos que el Mercosur, y casi con la misma influencia que las dinámicas sudamericanas, donde el actor de mayor peso es Brasil también.

Gráfico 1. Influencia de dinámicas regionales o globales. Por partido de pertenencia.

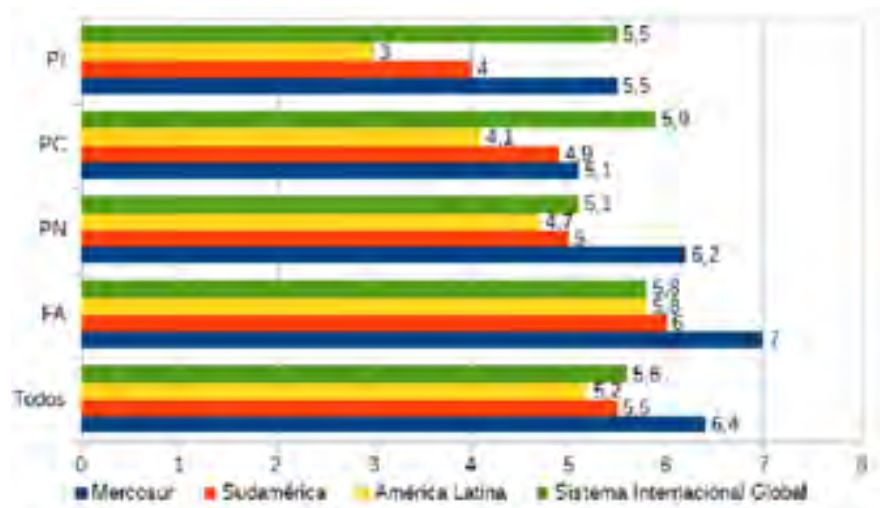

Fuente: Elaboración propia a partir de encuesta realizada a legisladores uruguayos.

\footnotetext{
${ }_{3}^{3}$ Para medir esta influencia se utilizó una escala donde 0 es ninguna influencia y 10 la máxima influencia posible.
} 
Para los frenteamplistas la dinámica del Mercosur es la que más influencia ejerce sobre la política exterior uruguaya, casi tanto como el papel que juega Brasil. La dinámica sudamericana ocupa el segundo lugar de influencia, al mismo nivel que Argentina si se observa la percepción de la influencia de los actores. Los procesos latinoamericanos y globales aparecen también con un peso relevante e igual entre los legisladores de este partido.

En el caso de las percepciones del Partido Nacional (PC) y el Partido Colorado (PC) se ve como las mismas son inversas. Mientras para el PN los procesos regionales (Mercosur) son los más influyentes seguidos por los globales (con aproximadamente un punto de distancia), para el PC las dinámicas globales son más relevantes que las del Mercosur. Tanto el PC como el PN colocan las dinámicas del espacio sudamericano en un tercer nivel de influencia en el entorno de la mitad de la escala. Para los legisladores del Partido Independiente (PI) la dinámica global y la del Mercosur tienen pesos relativos iguales.

\section{Entre Argentina y Brasil}

En la Tabla 2 se presenta la mirada de los parlamentarios sobre las relaciones Uruguay-Brasil y Uruguay-Argentina, comparando el fin de los gobiernos de coalición de los partidos fundacionales (PC y PN) y el gobierno de José Mujica y la visión prospectiva sobre el futuro de dichas relaciones. En concreto se puede observar que con respecto al futuro de las relaciones con el Brasil tres de cada cinco la vislumbran mejor en la próxima década, y casi un cuarto igual. Esta mirada prospectiva muy positiva sobre las relaciones con Brasil es, incluso, un poco mejor entre los legisladores frenteamplistas para los cuales serán mejores para casi dos tercios de los mismos e iguales para un quinto, con un $15 \%$ que no sabe o no está seguro de cómo van a evolucionar.

Mientras que para los legisladores del PN las perspectivas de mejora son la mayoría absoluta, con tres de cada diez viendo las relaciones en términos similares a los actuales y un $16,7 \%$ que no emite opinión. Los legisladores colorados tienen una posición intermedia con respecto a los dos anteriores, con tres de cada cinco viendo relaciones con Brasil mejores en el futuro y otro quinto igual. Los dos legisladores del PI ven las relaciones mejorando en la próxima década.

Las perspectivas reseñadas si bien son mejores que las que se tienen con respecto a Argentina no lo son mucho más, dado que la diferencia sólo alcanza los cuatro puntos porcentuales para el total de legisladores; similar porcentaje entre los frenteamplistas, dos puntos a favor de una mayor mejoría con la Argentina entre los nacionalistas y en cambio ocho puntos más a favor de Brasil entre los colorados. 
Tabla 2. Evaluación y prospectiva de las relaciones de Uruguay con Brasil y Argentina a una década por partido político de pertenencia.

\begin{tabular}{|c|c|c|c|c|c|c|c|c|c|}
\hline & & \multicolumn{4}{|c|}{ Argentina } & \multicolumn{4}{|c|}{ Brasil } \\
\hline & & \multicolumn{2}{|c|}{$\begin{array}{l}\text { Con respecto a } \\
\text { hace diez años }\end{array}$} & \multicolumn{2}{|c|}{$\begin{array}{l}\text { Dentro de } \\
\text { diez años }\end{array}$} & \multicolumn{2}{|c|}{$\begin{array}{l}\text { Con respecto a } \\
\text { hace diez años }\end{array}$} & \multicolumn{2}{|c|}{$\begin{array}{l}\text { Dentro de } \\
\text { diez años }\end{array}$} \\
\hline & & Frec. & $\%$ & Frec. & $\%$ & Frec. & $\%$ & Frec. & $\%$ \\
\hline \multirow[t]{5}{*}{$\begin{array}{l}\text { Todos los } \\
\text { legisladores }\end{array}$} & Mejor & 38 & 30,4 & 72 & 57,6 & 79 & 63,2 & 77 & 61,6 \\
\hline & Igual & 20 & 16,0 & 23 & 18,4 & 41 & 32,8 & 28 & 22,4 \\
\hline & Peor & 64 & 51,2 & 3 & 2,4 & 3 & 2,4 & & \\
\hline & $\mathrm{NS} / \mathrm{NC}$ & 3 & 2,4 & 27 & 21,6 & 2 & 1,6 & 20 & 16,0 \\
\hline & Total & 125 & 100 & 125 & 100 & 125 & 100 & 125 & 100 \\
\hline \multirow[t]{5}{*}{ FA } & Mejor & 34 & 51,5 & 40 & 60,6 & 62 & 93,9 & 43 & 65,2 \\
\hline & Igual & 12 & 18,2 & 13 & 19,7 & 4 & 6,1 & 13 & 19,7 \\
\hline & Peor & 19 & 28,8 & & & & & & \\
\hline & $\mathrm{NS} / \mathrm{NC}$ & 1 & 1,5 & 13 & 19,7 & & & 10 & 15,1 \\
\hline & Total & 66 & 100 & 66 & 100 & 66 & 100 & 66 & 100 \\
\hline \multirow[t]{5}{*}{ PN } & Mejor & 3 & 8,3 & 20 & 55,5 & 12 & 33,3 & 19 & 53,6 \\
\hline & Igual & 5 & 13,9 & 6 & 16,7 & 30 & 55,5 & 11 & 30,6 \\
\hline & Peor & 27 & 75,0 & 1 & 2,8 & 3 & 8,3 & & \\
\hline & $\mathrm{NS} / \mathrm{NC}$ & 1 & 2,8 & 9 & 25,0 & 1 & 2,8 & 6 & 16,7 \\
\hline & Total & 36 & 100 & 36 & 100 & 36 & 100 & 36 & 100 \\
\hline \multirow[t]{5}{*}{$\mathrm{PC}$} & Mejor & 1 & 4,8 & 11 & 52,4 & 5 & 23,8 & 13 & 61,9 \\
\hline & Igual & 3 & 14,3 & 4 & 19,1 & 15 & 71,4 & 4 & 19,1 \\
\hline & Peor & 16 & 76,2 & 2 & 9,5 & & & & \\
\hline & $\mathrm{NS} / \mathrm{NC}$ & 1 & 4,8 & 4 & 19,1 & 1 & 4,8 & 4 & 19,1 \\
\hline & Total & 21 & 100 & 21 & 100 & 21 & 100 & 21 & 100 \\
\hline \multirow[t]{5}{*}{ PI } & Mejor & & & 1 & 50 & & & 2 & 100 \\
\hline & Igual & & & & & 2 & 100 & & \\
\hline & Peor & 2 & 100 & & & & & & \\
\hline & $\mathrm{NS} / \mathrm{NC}$ & & & 1 & 50 & & & & \\
\hline & Total & 2 & 100 & 2 & 100 & 2 & 100 & 2 & 100 \\
\hline
\end{tabular}

Fuente: Elaboración propia a partir de encuesta realizada a legisladores uruguayos.

Mientras que para los legisladores del PN las perspectivas de mejora son la mayoría absoluta, con tres de cada diez viendo las relaciones en términos similares a los actuales y un $16,7 \%$ que no emite opinión. Los legisladores colorados tienen una posición intermedia con respecto a los dos anteriores, 
con tres de cada cinco viendo relaciones con Brasil mejores en el futuro y otro quinto igual. Los dos legisladores del PI ven las relaciones mejorando en la próxima década.

Las perspectivas reseñadas si bien son mejores que las que se tienen con respecto a Argentina no lo son mucho más, dado que la diferencia sólo alcanza los cuatro puntos porcentuales para el total de legisladores; similar porcentaje entre los frenteamplistas, dos puntos a favor de una mayor mejoría con la Argentina entre los nacionalistas y en cambio ocho puntos más a favor de Brasil entre los colorados.

Muy distinta es evaluación de situación con respecto a la situación que se daba en el 2005, puesto que mientras casi dos terceras partes de los legisladores la ven como mejor con respecto al Brasil y el restante tercio igual, la mitad ve la relación con Argentina como peor con respecto a hace diez años y sólo tres de cada diez mejor. La mejoría en las relaciones con Brasil es compartida por casi todos los frentistas (el 93,9\%), un tercio los blancos, y un cuarto de los colorados, primando entre los legisladores de los partidos tradicionales la idea de que la relación es similar a la que se tenía al comienzo de los gobiernos frenteamplistas $(55,5 \%$ y 71,4\% de blancos y colorados piensan así respectivamente).

Con respecto a la Argentina más de la mitad de los legisladores oficialistas ven una mejora en las relaciones durante el gobierno de su partido, opinión que no es compartida por los legisladores de la oposición entre los cuales tres de cada cuatro la ven incluso peor a como la dejaron ellos al abandonar el gobierno y por tanto la conducción de la política exterior en general y las relaciones con Argentina y Brasil en particular.

Luego de analizar los datos presentados, podría señalarse como la izquierda (FA) priorizaba a la región y a Brasil y el resto de los países emergentes como los aliados estratégicos. A nivel intrapartidario el posicionamiento entre sus alas ideológicas mostraba matices. Su ala izquierda presentaba un apoyo mayor a la región y a la apuesta por Brasil y el resto de los países emergentes como aliados estratégicos, mientras que el ala derecha del partido dividía sus posiciones en mitades que apoyaban la priorización de la región o el equilibrio entre la región y el mundo, teniendo una visión donde los aliados estratégicos varían según la agenda, aunque Brasil y los países emergentes es la segunda preferencia en este caso.

A la derecha del sistema de partidos uruguayo, los partidos fundacionales mayoritariamente están a favor de la apertura al mundo, considerando que los aliados estratégicos varían según los temas de la agenda. Aunque en el caso del PC, posicionado más a la derecha y con una tradición panamericanista 
fuerte, puede verse una tendencia de considerar a EEUU y el resto de los países desarrollados como aliados estratégicos. La segunda preferencia del ala derecha del PC en lo que refiere a aliados estratégicos es la antes señalada. Por su parte, en el PN, ubicado a la izquierda del PC - tomando en cuenta las medias de cada partido-, la segunda preferencia es Brasil y el resto de los países emergentes. Desde la oposición un senador manifestó: "Un Brasil líder regional debería ser como Alemania en la Unión Europea, debería brindar asistencia a los otros países".

En la mirada de los partidos políticos uruguayos parece existir un aspecto vinculado a las afinidades ideológicas o concebirse como parte de una familia política (caso del Partido dos Trabalhadores [PT] y el FA) que condiciona positiva o negativamente la relación, en función de cuál sea el partido de gobierno. Esto hace a que algunos actores políticos se cuestionen la alianza estratégica con Brasil a partir de la incertidumbre que puede generar una rotación política en el gobierno brasileño. Derechas e izquierdas acuerdan sobre la importancia de Brasil, pero es probable que estén pensando en "brasiles" distintos. El ala izquierda de la izquierda ve en Brasil al potencial líder regional y prefiere un proceso de integración regional profundo donde se avance más allá de la dimensión comercial. Por otra parte, el ala derecha de la izquierda ve en Brasil un líder regional, pero en un escenario de regionalismo abierto, donde lo central es la dimensión económico-comercial y la región es una plataforma de proyección al mundo. Por otra parte, los partidos de derecha (PC y PN) ven en Brasil a un el actor global, un socio privilegiado que podría operar como un "puente al mundo" para la inserción internacional de Uruguay.

El Brasil de Temer ya no es el Brasil del PT. La izquierda uruguaya que apostaba al liderazgo brasileño construido en tiempos de Lula y Celso Amorim hoy se encuentra en otro escenario. Mientras el nuevo gobierno brasileño parece lograr convergencias con la Argentina de Macri, la oposición uruguaya ( $\mathrm{PN}, \mathrm{PC}$ y $\mathrm{PI}$ ) valora positivamente los cambios en la región y particularmente la búsqueda de dar mayor dinamismo a la agenda externa del Mercosur. El partido de gobierno (FA), con preferencias divergentes en política exterior, encuentra un escenario donde uno de los debates es el margen de maniobra posible para el país. El cambio en la región constriñe la política exterior uruguaya a la vez que favorece una orientación que es valorada positivamente por la oposición y por el ala moderada del partido de gobierno. Mientras tanto el EEUU de Trump se mantiene distante de la región, se retira de los procesos de negociación plurilaterales de comercio y China continúa avanzando estratégicamente sobre América Latina. 


\section{Algunas reflexiones finales}

En el marco de una fuerte recesión económica y una crisis política interna en proceso, Brasil puede ver sensiblemente afectada la orientación de su política exterior; es altamente probable que los cambios en su política interna impacten sobre su política exterior. Ello en un contexto en el cual se desarrolla una competencia por la primacía en la región latinoamericana, con el avance de China, el posicionamiento de México y el reciente reperfilamiento de Argentina que, a partir del cambio de gobierno del año 2015, emerge como un potencial aliado estratégico de EEUU, aunque este escenario fue negativamente afectado por la asunción de Trump y sus lineamientos de política exterior.

En el caso específico de Uruguay los cambios que se están procesando en la región en general y en el Brasil en particular llevan a retomar las interrogantes ya planteadas en el cuerpo del trabajo: ¿se mantendrá en los próximos años la cercanía con Brasil por parte de la política exterior uruguaya? Al respecto se puede afirmar que durante la primera mitad del actual gobierno la relación con Brasil ha sido buena pero con una gestualidad mucho más sobria que la del pasado para con los gobiernos de Lula y Dilma, pero también de menor intensidad de la que se puso en marcha con la Argentina de Mauricio Macri, por lo que no sólo incide el cambio de signo político en el gobierno brasileño, sino también afinidades personales y, porque no, juicios sobre la legitimidad del desplazamiento de la presidenta Rousseff, nunca cuestionado oficialmente por Uruguay pero que si ha sido cuestionado por fuerza política Frente Amplio sustento político-partidario del gobierno nacional uruguayo. ¿O la alianza estratégica fue simplemente un movimiento táctico y coyuntural para aprovechar las ventajas de la coyuntura o se mantendrá en el mediano y largo plazo? La respuesta es que se dará en el largo plazo por razones geopolíticas, históricas y económico-comerciales. Pero la concreción de dicha alianza tiene como condición necesaria el reposicionamiento de Brasil como actor global y líder regional. No hay alianza conveniente para Uruguay sin atractivo y capacidades de seducción puestas en juego por parte de Brasil; en el mediano plazo otros cantos de sirenas pueden perfectamente sustituir el llamado brasileño y no habrá, posiblemente, mástil de la integración al que aferrarse con firmeza si todo continua como hasta ahora.

Como resultado de su profunda crisis política y sus dificultades económicas Brasil podría bajar el perfil de su política exterior o hacerla tributaria de otras potencias mundiales. Ante la debilidad brasileña el atractivo para los otros países de la región de un posible liderazgo benigno se devalúa y tiende a verse, por parte de algunos de sus vecinos y socios en la región, como un espejismo, 
no como una posibilidad realizable. La disminución del peso internacional brasileño o su supeditación a otra $\mathrm{u}$ otras políticas exteriores son posibles opciones de política pública y, en tanto tal, serán fruto de una construcción política, económica, social y cultural que procesarán los sistemas políticos y las sociedades de la región, no sólo el sistema político y la sociedad brasileños.

Asistimos a un momento de naturaleza bidireccional, que va de Brasil a los países de la región y de los países de la región al Brasil: ¿qué hará Brasil con su política exterior, en el nivel regional y mundial?, ¿contribuirán los países latinoamericanos a la construcción de un sistema de liderazgo en el cual todo el peso y los costos no recaigan sobre Brasil?

\section{Referencias}

ALMEIDA, Paulo Roberto de; BARBOSA, Rubens Antônio (orgs.). Relações BrasilEstados Unidos: assimetrias e convergências. São Paulo: Saraiva, 2006.

ALVARIZA, Rafael; CLEMENTE, Isabel. Las relaciones Uruguay-Brasil y la cooperación bilateral desde una perspectiva uruguaya. Revista Latino-Americana de História, v. 12, n. 3, p. 153-175, 2014.

AMORIM NETO, Octavio. De Dutra a Lula: a condução e os determinantes da política externa brasileira. Rio de Janeiro: Elsevier, 2012.

BURGES, Sean. Revisiting consensual hegemony: Brazilian regional leadership in question. International Politics, v. 52, n. 2, p. 193-207, 2015.

CAETANO, Gerardo. A cien años de la muerte del barón de Río Branco: la contemporaneidad del tratado de rectificación de límites en el río Yaguarón y la Laguna Merim (1909-1910). Cuadernos del Claeh, v. 100, n. 1, p. 105-123, 2012.

CAETANO, Gerardo; LÓPEZ BURIAN, Camilo; LUJÁN, Carlos. La política exterior uruguaya entre Vázquez y Vázquez (2010-2015). En: José Busquets y Nicolás Bentancur (coords.). El decenio progresista. Las políticas públicas de Vázquez a Mujica. Fin de Siglo: Montevideo, 2016. p. 279-300.

CERVO, Amado. Política exterior e relações internacionais do Brasil: enfoque paradigmático. Revista Brasileira de Política Internacional, v. 46, n. 2, p. 5-25, 2003.

CERVO, Amado. Política exterior do Brasil: o peso da História. Plenarium, v. 2, n. 2 , p. 10-26, 2005.

DOSCH, Jörn; GOODMAN, David. China and Latin America: complementarity, competition, and globalisation. Journal of Current Chinese Affairs, v. 41, n. 1. p. 3-19, 2012.

FLEMES, Daniel. Brazilian foreign policy in the changing world order. South African Journal of International Affairs, v. 16, n. 2, p. 161-182, 2009.

FLEMES, Daniel; WOJCZEWSKI, Thorsten. Contested leadership in international relations: power politics in South America, South Asia and Sub-Saharan Africa. Hamburg: Giga Working Paper n. 121, 2010. 
FLEMES, Daniel; WEHNER, Leslie. Drivers of strategic contestation: the case of South America. International Politics, v. 52, n. 2. p. 163-177, 2015.

FONSECA, Gelson. A legitimidade e outras questões internacionais. São Paulo: Paz e Terra, 2004.

HIRST, Monica. Brasil-Estados Unidos: desencontros e afinidades. Rio de Janeiro: FGV, 2009.

HURRELL, Andrew. An emerging security community in South America? En: Emanuel Adler; Michael Barnett (eds.). Security communities. Cambridge: Cambridge University Press, 1998. p. 228-264.

JENKINS, Rhys. Chinese competition and Brazilian exports of manufactures. Oxford Development Studies, v. 42, n. 3. p. 395-418, 2014.

LIMA, Maria Regina Soares de. Ejes analíticos y conflicto de paradigmas en la política exterior brasileña. América Latina/Internacional, v. 1, n. 2, p. 27-46, 1994.

LÓPEZ BURIAN, Camilo. Los partidos políticos uruguayos y la relación UruguayBrasil (2010-2013). En: Maria Izabel Mallmann; Tereza Cristina Schneider Marques (coords.). Fronteiras \& Relações Brasil-Uruguai. Porto Alegre: Edipucrs, 2015. p. 85-108.

LÓPEZ BURIAN, Camilo. Los impactos de la crisis de Brasil en la política exterior de Uruguay (2015-2016). Conjuntura Austral. Journal of the Global South, v. 7, n. 37, p. 25-39. 2016.

LUJÁN, Carlos. Brazil as a global stakeholder: a view from Uruguay. En: María Herminia Tavares de Almeida; Gian Luca Gardini (coords.). Foreign policy responses to the rise of Brazil: balancing power in emerging states. Londres: Palgrave Macmillan, 2016. p. 90-113.

MALAMUD, Andrés. A leader without followers? The growing divergence between the regional and global performance of Brazilian foreign policy. Latin American Politics and Society, v. 53, n. 3. p. 1-24, 2011.

MONIZ BANDEIRA, Luiz. Continuidade e mudança na política externa brasileira. Revista Brasileira de Política Internacional, v. 24, n. 2, p. 91-98, 1986.

MONIZ BANDEIRA, Luiz. Brasil, Argentina e Estados Unidos: da Tríplice Aliança ao Mercosul. Rio de Janeiro: Revan, 2003.

MONIZ BANDEIRA, Luiz. As relações perigosas: Brasil-Estados Unidos (de Collor a Lula). Rio de Janeiro: Civilização Brasileira, 2004.

MONIZ BANDEIRA, Luiz. Brasil, Argentina e Estados Unidos: conflito e integração na América do Sul. Rio de Janeiro: Civilização Brasileira, 2010.

ONUKI, Janina; OLIVEIRA, Amâncio de. Eleições, partidos políticos e política externa no Brasil. Revista Política Hoje, v. 19, n. 1, p. 144-185, 2010.

PÉREZ, Romeo. Política exterior uruguaya. Montevideo: Ediciones de la Plaza, 2011. PINHEIRO, Leticia; GAIO, Gabrieli. Cooperation for development, Brazilian regional leadership and global protagonism. Brazilian Political Science Review, v. 8, n. 2, p. 8-30, 2014. 
PINHEIRO, Leticia. Traídos pelo desejo: um ensaio sobre a teoria e a prática da política externa brasileira contemporânea. Contexto Internacional, v. 22, n. 2, p. 305-336, 2000.

PINHEIRO, Leticia. Politica externa brasileira. Río de Janeiro: Jorge Zahar, 2004.

RODRÍGUEZ, Júlio César Cossio. A política externa brasileira. En: Lúcia Avelar y Antônio Octàvio Cintra (orgs.). Sistema político brasileiro: uma introdução. 3. ed. São Paulo: Konrad Adenauer Stiftung, 2015. p. 373-388.

SARAIVA, Miriam Gomes. Brazilian foreign policy towards South America during the Lula Administration: caught between South America and Mercosur. Revista Brasileira de Política Internacional, v. 53, n. 1, p. 151-168, 2010.

SARAIVA, Miriam Gomes; TEDESCO, Laura. Argentina y Brasil. Políticas exteriores comparadas tras la Guerra Fría. En: Vicente Palermo (comp.). Politica brasileña contemporánea: de Collor a Lula en años de transformación. Buenos Aires: Siglo XXI, 2004. p. 475-512.

SILVA, André Luiz Reis da; SPOHR, Alexandre Piffero. Os percalços ao diálogo estratégico: as relações entre o Brasil e os Estados Unidos desde 2003. Monções, v. 4, n. 7, p. 69-91, 2015.

STRÜVER, Georg. 'Bereft of Friends'? China's rise and search for political partners in South America. Chinese Journal of International Politics, v. 7, n. 1, p. 117-51, 2014.

TUSSIE, Diana; COMINI, Nicolás. Argetina: reconfigurando el regionalismo en la ola conservadora. Informe de Coyuntura, n. 21, Montevideo: Orlac, 2016.

URDINEZ, Francisco. The political economy of the Chinese market economy status given by Argentina and Brazil. CS, v. 14, n. 1, p. 47-75, 2014.

URDINEZ, Francisco; LÓPEZ BURIAN, Camilo; OLIVEIRA, Amâncio de. Mercosur and the Brazilian leadership challenge in the era of Chinese growth: a Uruguayan foreign policy perspective. New Global Studies, v. 10, n. 1. p. 1-25, 2016.

VIGEVANI, Tullo; FAVARON, Gustavo de Mauro; RAMANZINI JÚNIOR, Haroldo; CORREIA, Rodrigo Alves. O papel da integração regional para o Brasil: universalismo, soberania e percepção das elites. Revista Brasileira de Política Internacional, v. 51, n. 1. p. 5-27, 2008.

Recibido el: 12 enero 2018

Aprobado el: 30 marzo 2018

Autor correspondiente:

Camilo López Burian

Constituyente 1502, Piso 6

1.200 Montevideo, Uruguay

CARLOS LUJÁN <clujan62@hotmail.com>

Doctor en Ciencia Política por la Universidad Católica del Uruguay (Montevideo, Uruguay), profesor e investgador de la Facultad de Ciencias Sociales de la Universidad de la República (Udelar, Montevideo, Uruguay).

ORCID: https://orcid.org/0000-0002-4742-6696

CAMILO LÓPEZ BURIAN <camilo.lopez@cienciassociales.edu.uy>

Doctor en Ciencia Política por la Universidad de la República (Udelar, Montevideo, Uruguay), profesor e investgador de la Facultad de Ciencias Sociales de la Universidad de la República (Udelar, Montevideo, Uruguay).

ORCID: https://orcid.org/0000-0003-1185-854X 